\title{
TOURISMUS UND VOLKSKUNDE ÜBERBLICK UND PERSPEKTIVEN DER KULTURWISSENSCHAFTLICHEN TOURISMUSFORSCHUNG
}

\author{
Adelheid SCHRUTKA-RECHTENSTAMM \\ Volkskundliches Seminar der Universität Bonn \\ D-53113 Bonn, Am Hofgarten 22, Deutschland
}

Die Urlaubsreise als Reise um des Reisens willen hat sich seit der Nachkriegszeit zum festen Bestandteil des Jahreslaufes entwickelt. Jährlich verreist immerhin fast die Hälfte der Bevölkerung Europas, und diejenigen, die noch nie in den Ferien unterwegs waren, werden immer weniger. Diese „schönsten Tage des Jahres“ (ARMANSKI 1978) stellen für viele Menschen die erwünschte und ersehnte Unterbrechung der Arbeitszeit dar, denen die Aufgabe zufällt, die alltäglichen Defizite in einer Vielzahl von Lebensbereichen auszugleichen.

Der Fremdenverkehr ${ }^{1}$ in seinem eigentlichen Wortsinn, als die Begegnung mit dem Unbekannten, gehört zu einem wichtigen Konstitutivum von Kultur als der kreativen Beschäftigung mit Neuem und Nicht-Alltäglichem und stellt einen wichtigen Beitrag zum durchaus brisanten Aspekt kultureller Kontakte und Konflikte dar (Vgl. GREVERUS u.a. 1988). In Vergangenheit und Gegenwart können eine Vielzahl von Reisearten und Mobilitätsvarianten beobachtet werden, man denke an Wallfahrten und Pilgerzüge, die Reisen der Handwerker oder Geschäftsleute, Forschungs- und Entdeckungsreisen, Migration, Ortsveränderungen durch Kriegswirren oder Vertreibung. Durch diese Reisen, denen die wirtschaftliche, politische oder religiöse Zweckgebundenheit gemeinsames Kennzeichen ist, kommt es zu Begegnungen mit anderen Regionen und Ländern. All dies sind Reisen, die das Forschungsinteresse der Volkskunde und verwandter Wissenschaften wecken, wie der Sammelband „Reisekultur“ eindrücklich zeigt (Vgl. BAUSINGER u.a. 1991)².

\section{TOURISMUSGESCHICHTLICHER ABRISS}

Als einer der Vorläufer des modernen Fremdenverkehrs gilt die Reise junger Adeliger durch Europa im 16. und 17. Jahrhundert. Ihre „Grand Tour“, die eine Art Initiationsritus in die feudale Gesellschaft darstellte, war Teil des adeligen Erziehungsprogramms und oft von mehreren Jahren Dauer und führte in die Metropolen

\footnotetext{
${ }^{1}$ Die Begriffe Fremdenverkehr und Tourismus werden synonym verwendet. nügen.

${ }^{2}$ Da diese Arten des Reisens nicht weiter berücksichtigt werden können, möge dieser Hinweis ge-
} 
des kulturellen, politischen und wirtschaftlichen Lebens. (Vgl. PRAHL-STEINECKE 1989). Gegen Ende des 18. Jahrhunderts wurde im Zuge gesellschaftlicher Umbrüche, durch die beginnende Industrialisierung und unter dem Einfluß der Aufklärung die Exklusivität dieser Reiseform aufgebrochen, und die neue Elite, das gehobene Bürgertum, reiste zu den kulturellen Zentren Europas. Die „Bildungsreise“ mit ihrer moralisch-pädagogischen Zielsetzung diente dem Kennenlernen kultureller, aber auch politischer, sozialer und technischer Einrichtungen. Eine große Zahl von realen und fiktiven Reisebeschreibungen und Reiseanleitungen (Apodemiken) erschien (Vgl. STAGL 1983), die mit ihren Schilderungen der Lebensumstände der Bevölkerung als volkskundliche Quellen interessant sind. Ihre Nachfolger waren die seit den 1830er Jahren erscheinenden Reiseführer - der erste Baedeker erscheint 1839 -, die durch ihre Beschreibungen und ihr Sternchensystem für besondere Sehenswürdigkeiten den touristischen Blick normierten. (Vgl. LAUTERBACH 1989 und 1993).

Im Zuge der beginnenden Romantik entstand die „sentimentale Reise“, die sich - vom humanistischen Bildungszweck befreit - zur touristischen Reise, der Reise als Selbstzweck, wandelte. Auch die Zielregionen wechselten. Seit der Mitte des 18. Jahrhunderts wurden im Sinne eines neuen Naturverständnisses Gegenden touristisch „entdeckt“, die bis dahin als unwirtlich, feindselig und ungesund gegolten hatten: die Meeresküste und die Berge. Adelige und Großindustrielle führten die Bäderreise zu den Meeresküsten und den binnenländischen Kurorten ein. Im Vordergrund standen dabei allerdings weniger die Gesundheit, sondern Vergnügungen und Aktivitäten wie die Promenade und der Besuch von Spielbank, Konzert oder Ball und die Treffen „der Gesellschaft“, wofür unter anderen Stefan Zweig oder Arthur Schnitzler noch um 1900 literarische Zeugnisse lieferten. Diese Prägung erwies sich als so stark, daß der Reise in die traditionellen Kurorte bis heute, da die Krankenkassen dort Erholungsbedürftige unterbringen, noch Reste des vergangenen Flairs anhaften.

Im damaligen Österreich-Ungarn, also der Donaumonarchie, standen die Böhmischen Bäder Karlsbad, Marienbad und Franzensbad und die Bäder an der Adria am Beginn der touristischen Entwicklung. Beliebt wurden auch der Schneeberg als Luftkurort, Baden bei Wien oder das Salzkammergut mit seinen Solebädern (BRUSATTI 1984: 14f). Bad Ischl und seine Umgebung verdankten ihre Bedeutung im 19. Jahrhundert der Vorliebe Kaiser Franz-Josefs, dort den Sommer zu verbringen. Ischl und das Salzkammergut avancierten in der zweiten Hälfte des 19. Jahrhunderts zum sommerlichen Treffpunkt bedeutender Politiker, Künstler, Adeliger und Großfinanziers. Man reiste „mit Wirtschaft“, das heißt mit Bediensteten und adäquater Ausstattung, und zum Teil mietete man sich auch in Häusern Einheimischer ein, wie dies Hugo von Hofmannsthal in seiner Erzählung „Das Dorf im Gebirge" so trefflich beschreibt. Aber auch der Bau von Villen und Landhäusern, die bis heute das Siedlungsbild im Salzkammergut prägen, wurde von diesen in Auftrag gegeben. (Vgl. OBERHAMMER 1983).

Die Einführung der Erfassung von Gästezahlen in den Kurorten ab dem Jahr 1875 dokumentiert die steigende Bedeutung des Fremdenverkehrs für Österreich- 
Ungarn ab der zweiten Hälfte des 19. Jahrhunderts. Spitzenreiter waren 1879 die böhmischen Kurorte gefolgt von Baden bei Wien, Bad Ischl, Bad Aussee, Wildbad Gastein, Bad Gleichenberg, Vöslau und Meran (BRUSATTI 1984: 30f.), wohingegen die heutigen österreichischen Tourismushochburgen in Tirol, Salzburg oder Vorarlberg noch kaum Bedeutung hatten.

Seit Anfang des 19. Jahrhunderts war es durch ein verändertes Naturverständnis und eine neue Naturwahrnehmung zur Entmythologisierung der Alpen, zu ihrer wissenschaflichen Erforschung und zu ersten touristischen Bergbesteigungen gekommen (Vgl. SEITZ 1987). Das Bild von den geheimnisvollen und schrecklichen Bergen, das jahrhundertelang vorgeherrscht hatte, wandelte sich in Bewunderung und Freude (OPASCHOWSKI 1989: 67). Entsprechend ihrer wirtschaftlichen Vormachtstellung waren es die Briten, die mit der Erschließung der Alpen begannen. Vornehmlich die Schweiz wurde zum Ziel des Stadtlebens überdrüssiger und naturschwärmerischer junger Reisender aus Großbritannien.

Bei der Entwicklung dieses Alpintourismus kam den Bergsteigerverbänden eine wichtige Bedeutung zu (Vgl. KRAMER 1983). Im Jahre 1857 war in London der erste „Alpine Club“ ins Leben gerufen worden. Fünf Jahre später folgte die Gründung des Österreichischen Alpenvereins, der ab 1873 als Deutsch-Österreichischer Alpenverein firmierte. Aus kulturwissenschaftlicher Sicht besonders interessant ist, daß neben der Förderung der Erforschung der Alpen, der Förderung des Bergsteigens und Wanderns und der Erhaltung der Schönheit und Ursprünglichkeit der Berge der Verein seine Aufgabe auch in der Pflege der Liebe zur deutschen Heimat sah (PRAHL-STEINECKE 1989: 50f.). In der Zeitschrift des Deutsch-Österreichischen Alpenvereins wurde neben Tourenbeschreibungen auch der Beschreibung der Lebensweise der alpinen Bevölkerung breiter Raum gelassen, die sich dadurch als ergiebiger und zum Teil kurioser Fundus erweist.

In der Folge derartiger aufklärerischer naturwissenschaftlicher Erkundungen kam es nicht nur zur Entdeckung der Landschaft, sondern es wuchs zunehmend das (binnen-)exotische und romantische Interesse am einfachen Leben der Einheimischen. Am Beispiel des Salzkammergutes läßt sich diese Entwicklung besonders deutlich darstellen. Durch die Übernahme von Kleidungsstücken durch die Touristen (Vgl. KRAJICEK 1979 und KAMMERHOFER-AGGERMANN-SCOPE 1994) und die Aufmerksamkeit für Bräuche wurden Teile der Alltagskultur der Bereisten durch den Tourismus gleichsam konserviert und erhielten eine neue Wertschätzung in veränderten Kontexten. Es ist sicherlich kein Zufall, daß zwischen Regionen mit frühem Fremdenverkehr und solchen, in denen die sogenannte „Volkskultur“ besonders reich vorhanden ist und gepflegt wird, zahlreiche Überschneidungen bestehen. Konrad Köstlins These, „,... daß der Tourismus das Land als kulturelles Phänomen erst geschaffen habe, als jene Region des anderen, des Fremden, in der die Exotik des Nahen wirksam wird“ (KÖSTLIN 1994: 9), erfährt hierdurch eine Bestätigung.

Die Untersuchung von Utz Jeggle und Gottfried Korff zum Regionalcharakter der Zillertaler gibt ein weiteres treffendes Beispiel für das Entstehen spezifischer Regionalklischees (JEGGLE-KORFF 1974). Wanderhändlern und reisenden Sängern aus dem Zillertal diente das Tirolertum im 19. Jahrhundert als „Verpackung“ und 
als Verkaufsstrategie und sie prägten damit das Bild vom „urigen“ Tiroler außerhalb des Landes. Diese Stereotype, die die Touristen vor Ort auch wiederfinden wollten, wurden von den Einheimischen verinnerlicht und Teil ihrer Identität.

Bis zur Mitte des 19. Jahrhunderts war die touristische Mobilität insgesamt noch gering, da breite Teile der Bevölkerung in ihren ökonomischen, rechtlichen und zeitlichen Möglichkeiten beschränkt waren. Eine der Grundbedingungen für die Entwicklung und zahlenmäßige Zunahme des Tourismus war die Bewußtmachung und Inwertsetzung von Freizeit im Gegensatz zu Arbeitszeit, die im 19. Jahrhundert ihren Anfang nahm. Bezahlter Urlaub wurde erst nach dem Ersten Weltkrieg nach und nach Bestandteil der Kollektivverträge. Auch hielt die beträchtliche Beschwerlichkeit des Reisens viele Menschen von derartigen Unternehmungen ab. Man reiste mit der Postkutsche (Vgl. BEYRER 1985), und auch die damaligen Straßen sind mit heutigen Verkehrsverbindungen kaum vergleichbar (Vgl. SCHARFE 1991). Völlig neue Möglichkeiten verbunden mit einem veränderten Distanzempfinden eröffnete die Einführung von Eisenbahn und Dampfschiff, die in der Lage waren, eine große Personenanzahl zu geringen Preisen und bei großer Geschwindigkeit zu transportieren. Damit konnte das gesamte Bürgertum zum neuen Reisepublikum werden (Vgl. SCHIVELBUSCH 1989).

Gegen Ende des 19. Jahrhunderts waren wesentliche Dimensionen des modernen Tourismus entwickelt: der Besichtigungs- und Bildungstourismus, der Kurfremdenverkehr, der Badetourismus, der Alpinismus und der Wintersporttourismus. Die gesellschaftliche Bewertung des Reisens war äußerst positiv. Das Reisen diente der Bildung, der Erfahrung und der Hebung von Status und Prestige, wobei die Parallelen zur heutigen Zeit evident sind. In diesen Jahren Zeit begannen auch Frauen verstärkt auf Reisen zu gehen (Vgl. JEDAMSKY u.a. 1994).

In der Donaumonarchie kam es um 1880 durch die steigenden Nächtigungszahlen zum Aufschwung des Fremdenverkehrs, der von da an auch von staatlicher Seite als neuer, eigenständiger Wirtschaftszweig erkannt wurde und dementsprechend gefördert werden sollte. Zu diesem Zweck lud der Grazer Fremdenverkehrsverein 1884 zum „Delegiertentag zur Förderung des Fremdenverkehrs in den österreichischen Alpenländern“. Der Fremdenverkehr wurde dabei als geeignetes Mittel erkannt, der zunehmenden Verarmung und Abwanderung in den ländlichen Regionen entgegenzuwirken (BRUSATTI 1984: 77). Ein weiteres Zeichen für die zunehmende Bedeutung des Fremdenverkehrs ist in der Aufnahme der Reisebüros in die Liste der konzessionierten Gewerbe Österreichs im Jahre 1895 (BRUSATTI 1984: 80) zu sehen. Die Idee des Reisebüros hatte seit der ersten Gründung 1845 durch den Engländer Thomas Cook (ENZENSBERGER 1962: 159) in ganz Europa Nachahmer gefunden.

Neben der Betonung der kulturellen Bedeutung des Fremdenverkehrs für die Volksbildung, wurden bereits kritische Stimmen laut, die vor sittlichen Gefahren und dem Verfall der Moral warnten (Vgl. GöTTSCH 1994). Die Kritik am Tourismus - so alt wie der Tourismus selbst - ist ein typisches Merkmal des Tourismus (Vgl. ENZENSBERGER 1962: 150f.).

Die touristische Form der Sommerfrische hatte um die Jahrhundertwende ihren 
Höhepunkt. Urlaubsregelungen und technologischer Fortschritt ermöglichten auch mittleren Beamten und Angestellten, Geschäftsleuten und Militärs mit ihren Familien die Reise aufs Land. Die Urlaubsreise zur Regeneration und Erholung von geistiger Arbeit (noch nicht von körperlicher Arbeit) hatte für diese neue Mittelschichten hohen Stellenwert und führte in noch weniger frequentierte und preiswertere Gebiete.

Nach dem Ende des Ersten Weltkrieges wurden die Verkehrsverbindungen verbessert und zahlreiche Seilbahnen (Vgl. TSCHOFEN 1994) gebaut. Der Fremdenverkehr der Zwischenkriegszeit läßt sich durch zunehmende Motorisierung, eine steigende Bedeutung der Wintersaison und die weiterhin sehr beliebte Erholungsform der Sommerfrische charakterisieren und weist auf die allmähliche Entwicklung des Tourismus zum Massenphänomen hin. Mit der Machtergreifung der Nationalsozialisten 1938 wurde der Fremdenverkehr politisiert und vereinnahmt, indem bisherige Fremdenverkehrsverbände durch nationalsozialistische Organisationen ersetzt und z.B. die organisierten „Kraft-durch-Freude-Fahrten“ eingeführt wurden (Vgl. u.a. KeRSCHBAUMER 1994; NUSSBAUMER 1988).

Kennzeichnendes Merkmal des Tourismus nach 1945 ist das Aufweichen sozialer Schranken durch die Teilnahme breiter Bevölkerungsgruppen am Tourismus. "Sich-erholen“ und die Urlaubsreise wurden zu einer wichtigen gesellschaftlichen Kategorie (ZimmermanN 1987: 109). Parallel zu dieser Entwicklung kam es zu einem massiven Ausbau der touristischen Infrastruktur, die zu markanten Veränderungen der Siedlungsstruktur in den Zielregionen führten. Der Fremdenverkehr in Mittel- und Westeuropa während der 60er und 70er Jahre wurde zu einem wichtigen Wirtschaftsfaktor und verhinderte durch die Schaffung von Einkunftsmöglichkeiten beispielsweise die weitere Zunahme der Landflucht. Der Fremdenverkehr erhielt als Arbeitgeber im wachsenden Hotel-, Gast- und Schankgewerbe, aber auch als Nebenverdienstsmöglichkeit in der Privatzimmervermietung und beim Urlaub am Bauernhof (Vgl. SCHRUTKA-RECHTENSTAMM 1992 und 1997a) verstärkte Relevanz.

Durch die steigende Bedeutung des Autos als Reisemittel veränderten sich nicht nur die Reisemodalitäten hin zu (vermeintlich) größerer Individualität. Der verstärkte Ausbau von Straßen führte zu einer Diffusion des Tourismus auch in abgelegene Regionen. Gleichermaßen entwickelt sich in den 50er und 60er Jahren der Campingurlaub mit seinen eigenen Gesetzmäßigkeiten als „Form der ungebundenen Freizeit- und Urlaubsgestaltung“ (HAAS 1993: 363).

Der Fremdenverkehr hatte in den letzten Jahrzehnten unter anderem vormals karge bergbäuerliche Lebensräume wie die alpine Landschaft in Wert gesetzt, und stagnierende Agrarräume entwickelten sich zu dynamischen Fremdenverkehrszentren (PREGLAU u.a. 1985: 29). Immer deutlicher rückten jedoch die Probleme, die der Massentourismus mit sich brachte, ins Bewußtsein der Betroffenen. Dazu gehört etwa die Konzentration des Fremdenverkehrs auf einige wenige Gemeinden - schon Ende der 60er Jahre wurden beispielsweise in Österreich 74 Prozent der Nächtigungen in 157, das sind ca. 5 Prozent, der Gemeinden des Landes gezählt (BRUSATTI 1984: 182). Die dichte Bebauung, die Zersiedelung der Landschaft und das Ansteigen des Transitverkehrs, aber auch die zunehmenden physischen und psychischen 
Belastungen der im Tourismus Beschäftigten sind Zeichen dieser Entwicklung (Vgl. PREGLAU u.a. 1985; SCHÖNBERGER 1994). Als Gegenstrategie tauchten in den 80er Jahren die Modelle vom ,anderen Reisen“, von der „Rücksicht auf Land und Leute“ und vom „sanften Tourismus“ auf ${ }^{3}$, die einen umwelt- und sozialverträglichen Fremdenverkehr propagieren, zum Schlagwort der 90er Jahre avancierte „Nachhaltigkeit“ (sustainability).

\section{FORSCHUNGSGESCHICHTLICHER ÜBERBLICK}

Gegen Ende des 19. Jahrhunderts, in der Zeit der Konsolidierung des Faches Volkskunde als Wissenschaft, läßt sich eine Übereinstimmung der Interessen von Touristen und Forschern an der bereisten regionalen Kultur feststellen. Als ein Beispiel $^{4}$ für die Ausrichtung der Volkskunde dieser frühen Stunde, die mit den Bestrebungen, das schwindende Erbe erfassen und retten zu wollen, mit den Vorstellungen von Touristen konform ging, verweise ich auf eine Notiz im ersten Jahrgang der Zeitschrift für österreichische Volkskunde aus dem Jahre 1895. Darin wird von einem stark beachteten Vortrag über „Die Bedeutung der Volkskunde und der Volkseigenthümlichkeiten für Touristik und Fremdenverkehr" berichtet, den eines der Mitglieder im Österreichischen Touristen-Club gehalten habe (Zeitschrift... 1895: 121f.). Es wird „als rettende Tat“ für die gefährdeten ,nationalen Besitztümer an Trachten, Sitten und Bräuchen" die Errichtung einer Sektion für Volkskunde im Touristen-Club empfohlen. Diese solle Anleitungen zur „Veranstaltung von Volksfesten und Volksspielen altherkömmlicher Art geben, um dadurch möglicherweise den Fremdenverkehr zu heben und zu beleben“. Diese Phase der Entstehung der Volkskunde als Wissenschaft ist durch Bestrebungen gekennzeichnet, „... Volkstümliches für irgendwelche Zwecke zu nützen, es zu pflegen und auch wiederzuerwecken, ..." (MOSER 1964: 31) und es damit auch gleichzeitig nach eigenen Vorstellungen zu verändern.

Die enge Verbindung von Forschung und Pflege verdeutlicht den gesellschaftspolitischen Auftrag, den sich die frühe Volkskunde gestellt hatte, die ganz dezidiert Kulturarbeit, Heimatpflege und Volksbildung als Forschungsziel formulierte (Vgl. GERAMB 1946).

Als Forschungsgegenstand selbst wurde der Fremdenverkehr von der Volkskunde jedoch erst in der Nachkriegszeit entdeckt. Durch seine funktionsbezogenen Forschungsansätze hatte Richard Weiss mit seiner „Volkskunde der Schweiz“ im Jahr 1946 einen wichtigen Neuanfang für die deutschsprachige Volkskunde gesetzt (Vgl. WEISS 1946). Er erkannte im Abschnitt über das Verkehrswesen den Einfluß des Fremdenverkehrs, der ,... in seinen großen Zentren das traditionelle Gepräge einer Gegend und das lokale Volksleben völlig verändert ..." (WEISS 1946: 119). Diese Berücksichtigung der jüngeren Entwicklungen und seine nuancierten Beobachtungen zu Veränderungen bedeuteten die Einbeziehung der Auswirkungen des Touris-

${ }^{3}$ Vgl. KRIPPENDORF u.a. (1988) mit ausführlicher Bibliographie zum Thema „Sanfter Tourismus“.

${ }^{4}$ Bei Hans Moser finden sich dafür zahlreiche Belege (MOSER 1964: 30-32). 
mus in die Aufgabenstellung der Volkskunde. Dies zeigt sich auch in seinen differenzierten Aussagen zu den Folgen des Fremdenverkehrs auf die Bereisten. Er merkte an, daß nach der ersten „Überflutung“ durch das Fremde die Besinnung der Einheimischen auf ihre Eigenart bewußt wieder erstanden ist (WEISS 1946: 120). Dennoch klingt hinsichtlich der Veränderungen durch den Tourismus immer wieder sein Bedauern durch, wenn er zum Beispiel von der „Prostitution des Volkslebens zum Schaustück für die Fremden“ (WEISS 1946: 119) sprach. Weiss thematisierte damit die Inszenierung von Kultur, die den Fremden von den Einheimischen geboten wird, und die ,... verschwindet, sobald man nach der Saison wieder daheim und unter sich ist." Neben den erwähnten pessimistischen Äußerungen zum Fremdenverkehr bedeutete für Weiss der Kontakt zwischen den Bergbewohnern und den Städtern aber auch das Entstehen von neuen Beziehungen, die er sogar als „willkommene Brücken“ (WEISS 1962: 239) bezeichnet. Sein Ziel lag primär darin, mögliche Verbesserungen der Lebensumstände der Bergbewohner aufzuzeigen, indem er die Tourismusindustrie mit anderen möglichen Erwerbsquellen verglich oder als schlechtere Alternative die Land- bzw. Bergflucht ansprach (WeISS 1962: 240).

Einen Zugang zur Thematik von anderer Seite findet Hans Moser, indem er die Rolle des Tourismus bei brauchgebundenen Darbietungen untersucht. Moser stellt bemerkenswerte Ansätze zur Diskussion, die in der Folge jedoch wie die von Weiss in der Forschung vorerst kaum Berücksichtigung fanden:

„Differenzierte Untersuchungen über das Reisevolk müßten auch die Volkskunde interessieren und mehr noch die Frage, wie das bodenständige Volk, das daran verdient und andrerseits nicht bloß materielle Leistungen bieten soll, reagiert und welche Veränderungen es durch diese Beanspruchung erfährt.“ (MOSER 1964: 42).

Im Zusammenhang mit dem Fremdenverkehr beschreibt Hans Moser das Entstehen von Phänomenen der Vermarktung von Volkskultur und brachte den Begriff „Folklorismus“ in die volkskundliche Diskussion ein. Der Folklorismus, für dessen bereits historisches Vorhandensein er zahlreiche Beispiele bringt, sei

„die Vorführung traditionell und funktionell festgelegter Elemente des Volkstums außerhalb ihrer lokalen oder ständischen Gemeinschaft, die spielerische Nachahmung volkstümlicher Motive in einer anderen Sozialschicht und das von verschiedenen Zwecken bestimmte Erfinden und Schaffen volkstümlich wirkender Elemente abseits der Tradition.“ (MOSER 1962: 190).

Auf einen kurzen Nenner gebracht, bezeichnet er derartige Entwicklungen als „Volkskultur aus zweiter Hand“ (MOSER 1962: 180). Einen wichtigen Unterschied des Folklorismus, wie er bis dahin zu beobachten war, zu dem am Beginn der 60er Jahre sieht er in dessen primär kommerziellen Bestimmung und seinem engen $\mathrm{Zu}$ sammenhang mit der Fremdenverkehrs- und der Unterhaltungsindustrie (MOSER 
1962: 199). Das Bedauern, das bei ihm ob dieser Phänomene, deren Untersuchung er als volkskundliche Forschungsaufgabe benennt, (MOSER 1962: 190) durchklingt, ist das Bedauern um den Verlust von Volkskultur.

Größere Bedeutung kommt dem Tourismus in Bausingers „Volkskunde“ als Forschungsgegenstand nicht nur unter dem Aspekt „Tourismus und Folklorismus“ zu (BAUSINGER [1971]). Bausinger deckt historische Hintergründe für die enge Verbindung von Folklorismus und Fremdenverkehr auf. Das Neue an diesen Überlegungen im Vergleich mit den Älteren von Weiss oder Moser ist nicht nur die Berücksichtigung der Entwicklung des Tourismus in seinen verschiedenen Phasen. Bausinger beschreibt Auswirkungen der Fremdenverkehrsindustrie auf Reisende und Einheimische, betont die spielerische Funktion des touristischen Folklorismus (BAUSINGER [1971]: 176) und demonstriert die Unwissenschaftlichkeit der Diskussionen um das „Echte“ in der Volkskultur. Bestimmte Rollenerwartungen und Verhaltensunsicherheiten sind seiner Meinung nach ebenso Bestandteil des Kontaktes zwischen Reisenden und Bereisten wie die Veränderung der regionalen Identität der Einheimischen.

Mit dem Ende der 70er Jahre erscheinen in der Volkskunde nur vereinzelt Studien zum Thema Tourismus. Exemplarisch sei hier das „Kritische Bilderbuch“ aus dem Frankfurter Institut für Kulturanthropologie und Europäische Ethnologie genannt (BEUTEL u.a. 1978). Das Buch geht über die bloße Kritik am Tourismus als Zerstörer von Kultur hinaus. Die komplexe Herangehensweise setzt den Tourismus selbst mit seinen ungleichen Partnern, dem Reisenden und dem Einheimischen, in den Mittelpunkt. Ina Maria Greverus hebt die Bedeutung, die dem Tourismus als Forschungsgegenstand beigemessen werden sollte, hervor, da seine Erforschung unter stark praxisorientierten Aspekten die „Schaffung der Voraussetzung einer partnerschaftlichen, und das heißt chancengleichen, interkulturellen Kommunikation“ (GREVERUS 1978: 97) vorantreiben könnte. Das Stichwort „praxisorientiert“ bedeutet auch, daß sich hier ein Berufsfeld für Absolventen kulturwissenschaftlicher Studien unter Zusammenarbeit mit der Tourismusbranche im Sinne einer Vermittlung von Lernangeboten an Reisende für eine ,prätouristische“ Reflexion eröffne (GREVERUS 1978: 97).

Eine intensive Hinwendung zu Möglichkeiten der Umsetzung volkskundlicher Forschung ist bei den Arbeiten von Dieter Kramer zu beobachten. Er hat sich seit Ende der 70er Jahre in zahlreichen Aufsätzen mit dem Tourismus und seinen gesellschaftspolitischen Hintergründen und Entwicklungen beschäftigt ${ }^{6}$. Seine Arbeit mit dem Titel „Der sanfte Tourismus“ (KRAMER 1983) ist nicht nur als Entwicklungsgeschichte des Alpinismus und seiner Vereine zu verstehen, sondern im Sinne einer praxisorientierten und verantwortungsvollen Wissenschaft diskutiert er negative Auswirkungen des Tourismus und mögliche Lösungswege für die Betroffenen.

Thematische Erweiterungen erfuhr die volkskundliche Tourismusforschung durch ein Projekt des Regensburger Instituts für Volkskunde unter der Leitung von

${ }^{6}$ Vgl. die Aufsatzsammlung „Tourismus-Politik“ (KRAMER 1990). 
Konrad Köstlin (BERWING-KÖSTLIN 1984). Neben der Erforschung einer Fremdenverkehrsregion, der Geschichte und Spezifik des Tourismus in Ostbayern, wurden neue Aspekte wie Andenken und Photographie oder das Essen auf Reisen behandelt. Damit wurden die Verhaltensweisen von Touristen in den Blickpunkt volkskundlicher Forschung gerückt.

Auch der Wiener Volkskundler Helmut P. Fielhauer äußerte sich zum Thema Fremdenverkehr und stellte seine Überlegungen zum Slogan der Österreichwerbung „Festland Österreich“ vor. Er betonte, daß die sogenannte Volkskultur und das Österreichbild durch den Tourismus geprägt werden und hob die Rolle, die dem Tourismus in Österreich zukommt, hervor. Bei der kulturellen Brisanz dieser Phänomene kritisiert er das Fehlen von volkskundlichen Untersuchungen zur Tourismuskultur und ihren Auswirkungen (FIELHAUER 1982). Als Stichwort - jedoch noch nicht als eigenen Forschungsgegenstand - hatte zwar Leopold Schmidt „Fremdenverkehr" in seine Bibliographie zur Volkskunde aufgenommen. Die volkskundlichen Aufsätze, die hier vor allem seit den 70er Jahren genannt werden, handeln allerdings vom - weil folkloristischen - meist negativen Einfluß des Tourismus auf Volkskultur bzw. es werden darin Arbeiten aus Nachbarwissenschaften wie Geographie oder den Wirtschafts- und Sozialwissenschaften angeführt (Bibliographie 1969ff.).

Die verstärkte Beschäftigung mit dem Phänomen Tourismus in der deutschsprachigen Volkskunde führte schließlich zur Gründung einer eigenen Kommission für kulturwissenschaftliche Tourismusforschung in der Deutschen Gesellschaft für Volkskunde im Jahre 1988. Zu Beginn ihrer Arbeit hatte sie es sich zum Ziel gesetzt, zunächst in einer Art Bestandsaufnahme spezifische Fragestellungen vorzustellen und darzulegen, wie sich die kulturwissenschaftlichen von den zahlreichen Forschungsansätzen der mit Tourismus befaßten Wissenschaften unterscheiden, um die diesbezügliche Lücke (KRAMER 1992: 11) schließen zu können. Die deutschsprachige Tourismusforschung war langezeit von einer Konzentration auf ökonomische Aspekte, auf Marktanalysen oder Tourismuskritik beschränkt, was auch Dieter Kramer und H. Jürgen Kagelmann monierten (KRAMER 1990; KAGELMANN 1993). Aufgabe einer kulturwissenschaftlichen Tourismusforschung muß es jedoch sein, eine differenzierte Sichtweise zu entwickeln und das negative Image, das beispielsweise dem Massentourismus anhaftet, zugunsten soziokultureller Fragestellungen aufzulösen und damit gesamtgesellschaftliche Bezüge miteinzubeziehen.

Für die Volkskunde bedeutet, wie oben dargelegt, der Tourismus ein relativ junges Forschungsfeld. In anderen Wissenschaften hat die Beschäftigung mit der Urlaubsreise schon weit längere Tradition, man denke an die Schweizer Wirtschaftswissenschaftler Walter Hunziker und Karl Krapf, die bereits zu Beginn der 40er Jahre ihre „Fremdenverkehrslehre“ verfaßten (HUNZIKER-KRAPF 1942) oder an die inzwischen zum Klassiker gewordene Untersuchung von Hans-Joachim Knebel über die soziologischen Strukturwandlungen im modernen Tourismus (KNEBEL 1960). Einen fachübergreifenden Einblick in die Disziplinen der Tourismuswissenschaft, ihre theoretischen Konzepte sowie Phänomene und Ausformungen des Tourismus gibt das Handbuch von Heinz Hahn und H. Jürgen Kagelmann (HAHNKAGELMANN 1993). 


\section{FORSCHUNGSANSÄTZE}

\section{DIE GASTGEBERKULTUR}

Der Tourismus nimmt Einfluß auf viele Bereiche der bereisten Kultur, die traditionellerweise volkskundliches Interesse hervorrufen und den alten Kanon repräsentieren, genannt seien Sitte und Brauch, Kleidung, Essen und Trinken oder Siedlung. Volkskundliche Tourismusforschung muß jedoch mehr sein als ein Anhängsel der „Kanonexerzitien“ (KRAMER 1992: 12). Es bedarf differenzierter Studien, um die Entwicklungen, die der Fremdenverkehr mit sich bringt, in ihren soziokulturellen Auswirkungen zu untersuchen und in ihrer Gesamtheit einer Analyse zu unterziehen. Wie oben dargelegt, wurden Veränderungen durch den Tourismus lange Zeit als folkloristisch und damit „unauthentisch“ abgetan. Es gilt jedoch die Gründe für die Suche nach dem „Echten“ und der Präsentation des „Echten“ bzw. dessen, was als „echt“ vermarktet wird, zu hinterfragen. Die Frage nach dem Authentischen wird damit sekundär (Vgl. BENDIX 1994).

Zum Folklorismus und zur Folklorismuskritik im Tourismus existieren bereits differenzierte Studien (Vgl. HörANDNER-LunZER 1982; BODEMANN 1983). Das Phänomen und der Begriff Folklorismus bleiben jedoch schwer entwirrbar, wofür Hermann Bausinger den ästhetischen Umgang mit Elementen der Volkskultur, den weiten räumlichen Ausgriff, die Dialektik von aktueller Lebendigkeit in der Gestaltung des Vorgeführten und musealen Implikationen und Zielen und schließlich das Nebeneinander und Ineinander von „Idealismus“ und kommerziellem Interesse als Gründe anführt (BAUSINGER 1988: 323f.). Ein jüngeres Beispiel der Beschäftigung mit den Zusammenhängen von Folklorismus und Tourismus stellt die Dissertation von Kriemhild Kapeller dar. Am Beispiel des Vorarlberger Fremdenverkehrs analysiert sie die vielschichtigen Wirkungsgrade des Folklorismus, von der Architektur über die Nahrung bis hin zur Werbung. (KAPELLER 1991).

Volkskundliche Tourismusforschung ist allerdings nicht auf Folklorismusforschung beschränkt. Ihre Aufgabe ist es darüberhinaus, Auswirkungen des Tourismus auf die soziokulturellen Bedingungen und Bedingtheiten, auf Verhaltensweisen und Werthaltungen der Bevölkerung in touristischen Zielgebieten zu untersuchen. Für die Bewohner von Fremdenverkehrsgebieten bildet die Präsenz von urlaubenden Menschen einen Bestandteil ihres Alltags. Ihre Einteilung des Jahres erfolgt je nach Anwesenheit von Touristen in Saison und Nicht-Saison, deren unterschiedliche Szenarien Orvar Löfgren eindrücklich beschrieben hat (LÖFGREN 1994).

In seinen Auswirkungen auf die Zielregionen wird der Fremdenverehr konträr einmal als Retter und einmal als Zerstörer interpretiert. Die Fremdenbeherbergung bringt Arbeitsplätze, wirtschaftlichen Aufschwung und Kontakte mit fremden Menschen, der kulturellen Welt der Gastgeber wird die "Chance zum Überleben“ (PREGLAU u.a. 1985: 105) geboten. Vor allem im ländlichen Umfeld wurde und wird der Fremdenverkehr bei wirtschaftlicher Stagnation oder Rezession von politischer Seite als Chance und Ausweg begriffen. Die Schaffung neuer Arbeitsplätze wird als Strategie gegen die Abwanderung Jugendlicher angeführt und die Förderung des 
Tourismus als Maßnahme dargestellt, von der jeder profitieren könne. Andererseits werden die Anforderungen an die Beschäftigten besonders im Massentourismus als stark belastend empfunden, wofür die Studie von Alwin Schönberger beredtes Zeugnis gibt, in der er exemplarisch Lebensläufe aus touristischen Milieus vorstellt (SCHÖNBERGER 1994). Die Erwartungen an das Engagement der Gastgeber steigen, was nicht ohne Folgen bleibt. ${ }^{8}$ Eine wichtige Quelle diesbezüglich stellt die Ratgeberliteratur für touristische Unternehmen dar, die neuerdings das Finden einer verträglichen Distanz und das Entwickeln einer spezifischen Identität als Gastgeber als wichtig erachtet, bei der es darauf ankomme, die geeignete Nähe und den notwendigen Abstand zu finden.

Verschiedentlich taucht Kritik an den mit dem Tourismus einhergehenden Veränderungen der Gastgeberkultur auf. Tendenzen zur Musealisierung und zur Käuflichkeit der Kultur und der drohende Verlust der kulturellen Eigenständigkeit werden als Nachteile beschrieben (PREGLAU u.a. 1985: 106f). Alternativmodelle sollen als „sanfter“ oder ,intelligenter" Tourismus die Bewahrung und die Erhaltung der bereisten Kultur miteinschließen. In der Kulturwissenschaft darf man derartige Aussagen nicht kritiklos übernehmen, da sie von einer zu statischen Betrachtungsweise zeugen angesichts einer bereits über hundertjährigen Geschichte des Tourismus in Europa. „Kritik an der Tourismuskritik“ von volkskundlicher Seite ist herausgefordert.

Veränderungen der Siedlungs- und Sozialstruktur durch Tourismus sind ganz offensichtlich. Gravierend und natürlich mit dieser in engem Bezug stehend, sind auch Veränderungen von Norm- und Wertvorstellungen, ohne diese von vornherein als negativ zu bezeichnen. Der Umbruch, den der Fremdenverkehr mit sich bringt, kann nur langsam verarbeitet werden und führt zu kulturellen Verunsicherungen. Er bringt beispielsweise eine Inwertsetzung des für die Gastgeberkultur Alltäglichen mit sich, eine „Folklorisierung des Alltags“, wie der Blick in die Reiseteile der Tagespresse bestätigt. Dabei können Verinnerlichungen der von außen herangetragenen Bilder beobachtet werden, die zu Veränderungen und schließlich zu Stabilisierungen der Identität führen (Vgl. BRUNER 1991; SCHRUTKARECHTENSTAMM 1995 und 1999).

\section{TOURISTENKULTUR UND REISEALLTAG}

Daß der Schweizer Volkskundler Ueli Gyr bei der Einführung der Termini „Touristenkultur“ und „Reisealltag“ auf deren scheinbare innere Widersprüchlichkeit hinweisen muß, zeigt, daß die Erforschung der touristischen Reise 1988 noch keinen ausgeprägten Forschungsgegenstand der Volkskunde darstellte. Die Ursachen dafür wähnt er einerseits in der pauschalen Kritik an den Reisenden und deren

${ }^{8} \mathrm{Zu}$ den Themen Widerstand und Strategien der Gastgeberkultur gegen die Veränderungen durch den Tourismus vgl. HAID (1988) und JOHLER (1994). 
massenhaften „kulturlosen“ Auftreten. Auf der anderen Seite wird der Urlaub als Gegenwelt ${ }^{9}$ zum alltäglichen Trott empfunden, sodaß die Verknüpfung von Alltag und Reise paradox erscheint. Gerade hier setzt kulturwissenschaftliche Forschung ein, da

„... der Urlaubsgestaltung (dem Reisen insbesondere) das Moment eigener Routinewirklichkeit, ritualisierter Aneignung von Fremdem und besonderer Techniken anhaftet, mit denen touristische Situationen bewältigt werden.“ (GYR 1988: 225).

Gyr legt in seinem programmatischen Aufsatz, dem er bezeichnenderweise den Untertitel „Volkskundlicher Nachholbedarf in der Tourismusforschung“ gibt, wichtige Grundlagen dar. An Beispielen des Fernreisens und des Cluburlaubs zeigt er die Sinnhaftigkeit von Mikrostudien auf, die dem stark ritualisierten touristischen Verhalten, das durch ausgeprägte Rollen- und Rhythmuswechsel gekennzeichnet ist, am ehesten gerecht werden.

Reisen als eine Bewegung im Raum bedeutet das Verlassen des vertrauten Territoriums und die Konfrontation mit dem Unvertrauten, dem Fremden, das bewältigt werden muß (EDER 1991: 158). Das Bestreben der meisten Reisenden ist es also, in der befremdlichen Umwelt einen Bereich zu finden, der Schutz bietet und Vertrauen schafft. Ein evidentes Zeichen dafür ist die Beliebtheit von Wohnwagen und Wohnmobil, (vgl. HOFMANN 1994), aber auch das Wiederkehren an vertraut gewordene Orte (Stammgastphänomen). Touristische Handlungen sind eng mit Ritualen und Symbolen verknüpft, die in diesem Sinne Sicherheit und Orientierung geben (SOEFFNER 1991). Einfache Deutungsmuster erleichtern dem Touristen, das Fremde und Neue als das Einfache und Übersichtliche zu erfahren und zu erleben (MACCANNELl 1989). Dabei tauchen ganz traditionelle volkskundliche Phänomene wie Berührungsmagie auf. Der Vergleich mit den Strukturen einer Wallfahrt drängt sich auf: die Souvenirs werden zu säkularisierten Devotionalien (GYR 1994 und SCHRUTKA-RECHTENSTAMM 1995).

Die touristische Aneignung von Fremdkulturellem erfolgt, wie bereits erwähnt, über Symbolkonsum: Sehenswürdigkeiten, zu denen die Reiseführer im Sternchenprinzip leiten, sind dafür evidente Beispiele (vgl. LAUTERBACH 1989 und 1993), der Kauf von Souvenirs (KÖSTLIN 1991) oder ausgewählten Verbrauchsgütern, das Schreiben von Ansichtskarten (LÖFGREN 1986, CANTAUW-GROSCHEK 1993), die symbolische Angleichung an das die Kultur des Urlaubslandes durch Kleidungsstücke oder Accessoirs oder die Konsumation von kulinarischen Spezialitäten und Getränken. Die Bilder der Ferne und des Urlaubs, die von den unterschiedlichsten Medien versprochen werden (WEYERS-KÖCK 1995), wollen vor Ort wiedergefunden werden, selbst dann, wenn die Touristen die Unmöglichkeit ihres Unterfangens durchschauen.

Der Urlaub wirkt aber auch in den Alltag hinein. Es gehören erwartungsvolle Vorbereitungen dazu: Pläne machen, Landkarten studieren und Prospekte lesen.

${ }^{9}$ Zur Kritik am Gegenweltmodell vgl. KöSTLIN (1995). 
Dazu zählen geradezu ritualisierte Handlungsabläufe wie das Kofferpacken. Auch in kanalisierten Erinnerungen in Form von Erzählungen oder in optischen Aufbereitungen wie den berühmt-berüchtigten Diaabenden werden Urlaub und NichtUrlaub miteinander verwoben. Der Symbolik dieser authentischen Erinnerungsbeweise haben Elisabeth Fendl und Klara Löffler Überlegungen gewidmet. (FENDLLÖFFLER 1995).

Obwohl oder eher weil Reisen ein Massenphänomen ist, läßt sich daran sehr deutlich soziales Differenzierungsstreben erkennen. Nicht mehr die Reise an sich bringt Prestige, sondern durch ein feinmaschiges Netz des Wohin und Wie schätzen sich die Touristen gegenseitig ein, sodaß Assoziationen zum Bourdieuschen Szenario der feinen Unterschiede nicht zufällig sind. Die verschiedenen Reiseformen mit dem Auto, Wohnmobil, Flugzeug oder mit dem Fahrrad, die organisierte Pauschalreise oder die altbewährte Sommerfrische ermöglichen nicht nur die soziale Zuordnung, sondern bestimmen auch die Intensität des Blickes auf das andere Land mit.

\section{TOURISMUS ALS KULTURKONTAKT}

„Wir sind alle Touristen“, wie dies Konrad Köstlin für den Titel eines Vortrages formulierte (KöSTLIN 1995) und - so könnte man ergänzen - wir sind alle Bereiste. Während aber die Begegnung für den Bereisten während seines Arbeitsalltags stattfindet, bedeuten sie für den Reisenden das „Außer-Alltägliche“, die kostbarsten Tage des Jahres. Berücksichtigt man, daß Freizeit höher bewertet wird als Arbeitszeit, so läßt sich auf das Vorhandensein gewisser hierarchischer Strukturen zwischen arbeitender und urlaubender Bevölkerung schließen, die im Extrem als „HerrKnecht-Beziehung" zu bewerten sind. Tourismus bedeutet das Aufeinandertreffen von Menschen unterschiedlicher kultureller, bzw. sozialer und regionaler Herkunft, aus der kulturelle Mißverständnisse bzw. kulturelles Nicht-Verstehen resultieren können. Es entwickelt sich ein diffiziles Geflecht von Status- und Machtverhältnissen, was der Soziologe Boas Shamir anhand des Trinkgeldgebens anschaulich dargestellt hat (SHAMIR 1993).

Heide Wahrlich hat in ihrer Untersuchung über den Ferntourismus die diesbezüglichen unterschiedlichen Intensitäten aufgezeigt: die flüchtige Kulturberührung, der konfliktreiche Kulturzusammenstoß, der Kulturkontakt, der ein ausgewogenes Kräfteverhältnis voraussetzt, die Kulturdurchdringung durch Akkulturation, die Kulturverflechtung als Ergebnis eines wechselseitigen Anpassungsprozesses und der Kulturschock bei sehr stark differierenden Kulturen, der große Wertunsicherheiten und Orientierungsschwierigkeiten mit sich bringt (WAHRLICH 1984: 43-49). $\mathrm{Zu}$ interaktiven Begegnungen zwischen Reisenden und Bereisten kommt es im allgemeinen nicht unmittelbar, sondern durch die Vermittlungsinstanz der Dienstleistung: Beherbergung, Verpflegung, Information, Verkauf oder Animation stellen Verknüpfungen zwischen Reisenden und Bereisten dar. Touristen treffen am Urlaubsziel auf Menschen des Gastgeberlandes in erster Linie in deren beruflicher Situation, reduziert auf ihre Rolle als Dienstleistende: als Hotelpersonal, Kellner, 
Animateure oder Verkäufer - ein Faktum, das bei der Analyse der Gast-Gastgeberbeziehungen weiterhin zu beachten ist. Marion Thiem trägt diesem Faktum Rechnung, indem sie vom Entstehen eigener touristischer Kulturen spricht: neben der Kultur des touristischen Zielgebietes, der Kultur des touristischen Quellgebietes und der Touristenkultur schließt ihr Ansatz eine tourismusspezifische Dienstleistungskultur mit ein (THIEM 1994).

Von Seiten der Gastgeber sind die Erwartungen funktional und von kommerziellem Interesse geprägt. Die Aufgabe der Anbieter von touristischen Leistungen ist es demnach, den Wünschen der Touristen gerecht zu werden. Damit können sie ihren wirtschaftlichen Erfolg maximieren und in ihrem Beruf erfolgreich sein. Die Zufriedenheit des Kunden ist eine Grundvoraussetzung für eine gelungene Dienstleistung. Gäste, die sich nicht wohl fühlen, kommen nicht wieder und sind schlecht für das Image. Der englische Soziologe Roy C. Wood beschäftigte sich in den Annals of Tourism Research mit Hotelkultur und erörterte die Erwartung der Gäste, daß die Dienstleistungen ihnen als Einzelpersonen, als private Individuen angeboten werden und nicht als anonymem Teil einer Masse (WoOD 1994: 71). Das Problem für das Hotel, wie für jedes touristische Unternehmen, ist es, für einen individuellen Service zu sorgen und gleichzeitig das Faktum zu verschleiern, daß es seine Bestimmung ist, eine generalisierende Geschäftsfunktion nicht an Individuen, sondern an die Masse, an alle Gäste, zu verteilen.

Welch wichtige Rolle die Betreuung durch die Gastgeber spielt, unterstreicht der Soziologe John Urry. Seine Beobachtung, daß gerade für Touristen, die immer wieder an den selben Ort reisen, das persönliche Engagement der Gastgeber wichtiger sei als das physische Ambiente, sprich: der Komfort und die Ausstattung des Hauses (URRY 1990: 71), wird in Interviews mit Stammgästen in kleinstrukturierten touristischen Unternehmen absolut bestätigt (SCHRUTKA-RECHTENSTAMM 1994 und 1997c). Gastgeber versuchen also, eine Atmosphäre herzustellen, in der sich ihre Gäste wohlfühlen. In diesem Sinne ist die Inszenierung des Wohlfühlens als Gefühlsmanagement bzw. Gefühlsarbeit, wie dies Arlie Russel Hochschild bezeichnet, als Instrumentalisierung von Gefühlen, als ihr bewußtes Einsetzen und ihre Kontrolle im beruflichen Kontext zu beschreiben (HOCHSCHILD 1990). Gefühlsarbeit hat ebenso wie die Leistungen, die über die Bezahlung hinaus erbracht werden, nicht nur im Tourismus, sondern in Dienstleistungsbereichen generell steigende Bedeutung.

Qualität, Service und Kundennähe sind die neuen Stichwörter, die in einer Fülle von theoretischen Analysen und praxisorientierten Ratgebern ihren Niederschlag finden (Vgl. u.a. ROMEIS-STRACKE 1995). Es handelt sich dabei um Werte, die mit der Gastfreundschaft als elementarem sozialen Prinzip, das die Aufnahme, den Schutz und die Bewirtung von Fremden bezeichnet, in Zusammenhang stehen. Gastfreundschaft existiert in seiner gegenwärtigen und vor allem historischen Unbestimmtheit nur als Idee, als hehres Prinzip. Diese „Idee der Gastfreundschaft“" schwingt auch in der gewerblichen Aufnahme von Reisenden mit. Sie kann einerseits als Teil der Erwartungen der Touristen dekodiert werden gemäß der Vorstellung, daß der Gast König sei und eine dementsprechende Aufnahme erfahren müsse. Das 
Gefühl, im Urlaub etwas zu erleben, zu erhalten, was jenseits der Bezahlung liegt, hat für reisende Urlauber seine besondere Bedeutung. Der Tourist wird zum willkommenen Gast, der Bereiste zum herzlichen Gastgeber und die Interaktion ist durch die Idee der Gastfreundschaft und ihre Symbolik geprägt, womit die Beziehung über die rein geschäftliche Basis hinausweist (vgl. SCHRUTKA-RECHTENSTAMM 1996).

Mit Slogans wie „Nicht Tourist, sondern Gast sein“ oder „Urlaub bei Freunden“ sollen die Urlauber erwarten, daß ihnen Gastfreundschaft entgegengebracht wird. Es werden Werte suggeriert, wie sie einer persönlichen und privaten Beziehung entsprechen. Die „Idee der Gastfreundschaft“ wird damit funktionalisiert und es entsteht eine spezifische „touristische Gastlichkeit“. Die Einführung der Termini "Gast" und „Gastgeber“" oder auch Gemütlichkeit und Freundlichkeit zielt gerade in der österreichischen Tourismuswerbung bewußt auf ein Image, das nicht kritik- aber auch nicht spurlos an den Bewohnern der Alpenrepublik vorbeigegangen ist (vgl. BRUCKMÜLLER 1994; MENASSE 1993).

Der Kontakt zwischen Reisenden und Bereisten ist geprägt durch gegenseitige Vorstellungen und Bilder. Fremd- und Autostereotype weisen jedoch große Differenzen auf, die, wie gezeigt wurde, auf unterschiedliche Prädispositionen zurückzuführen sind. Diese Diskrepanz erfordert die Herausbildung von dem Tourismus immanenten, eigenen Ritualisierungen, die die Begegnung kalkulierbar machen und Orientierungssicherheit gewährleisten. Das Interesse der kulturwissenschaftlichen Tourismusforschung schließt bei der Dekodierung dieser Zeichensysteme und Prozesse auch kulturspezifische Hintergründe und gesamtgesellschaftliche Bezüge mit ein (vgl. SCHRUTKA-RECHTENSTAMM 1997c).

\section{LITERATUR}

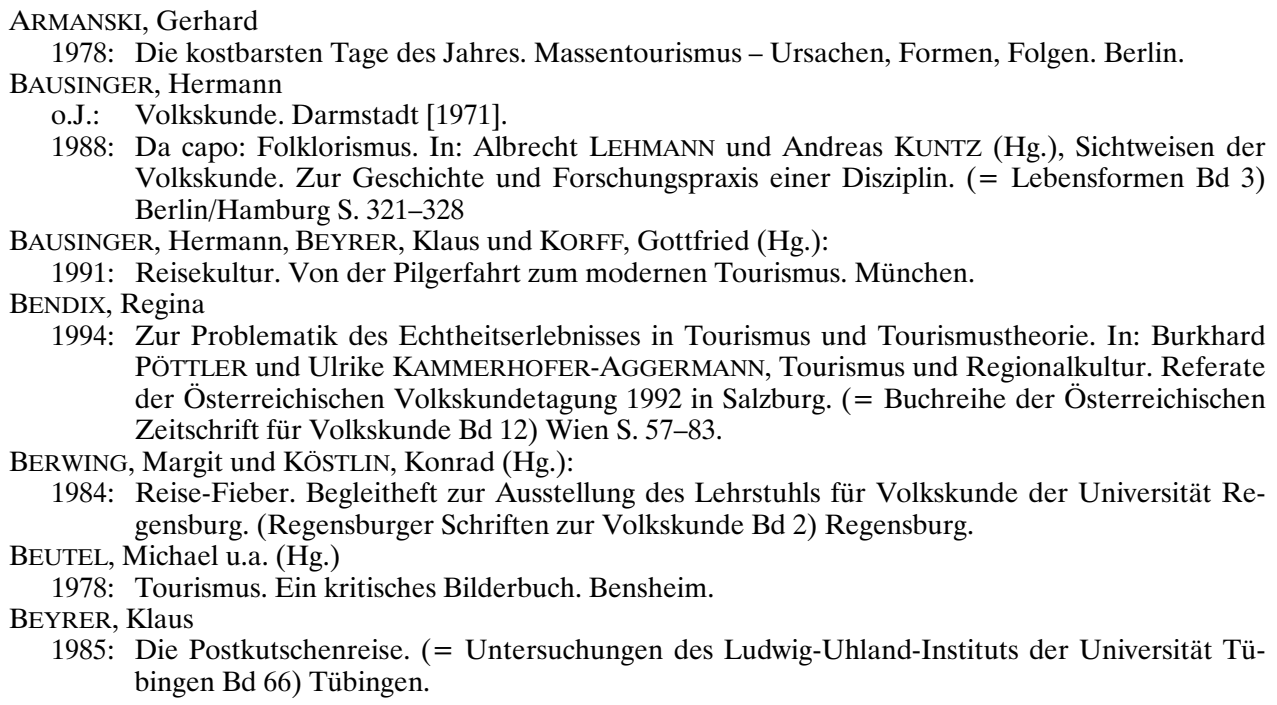

1994: Zur Problematik des Echtheitserlebnisses in Tourismus und Tourismustheorie. In: Burkhard PÖTTLER und Ulrike KAMMERHOFER-AGGERMANN, Tourismus und Regionalkultur. Referate der Österreichischen Volkskundetagung 1992 in Salzburg. (= Buchreihe der Österreichischen Zeitschrift für Volkskunde Bd 12) Wien S. 57-83.

BERWING, Margit und KöstLIN, Konrad (Hg.):

1984: Reise-Fieber. Begleitheft zur Ausstellung des Lehrstuhls für Volkskunde der Universität Regensburg. (Regensburger Schriften zur Volkskunde Bd 2) Regensburg.

1985: Die Postkutschenreise. (= Untersuchungen des Ludwig-Uhland-Instituts der Universität Tübingen Bd 66) Tübingen. 
Bibliographie, österreichische volkskundliche 1969ff: Verzeichnis der Neuerscheinungen. Hg.: Klaus BEITL, Wien.

BODEMANN, Ulrike

1983: Folklorismus - ein Modellentwurf. In: Rheinisch-Westfälische Zeitschrift für Volkskunde. Jg. 28, S. 101-110.

BRUCKMÜLLER, Ernst

1994: Österreichbewußtsein im Wandel. Idenität und Selbstverständnis in den 90er Jahren. Wien.

BRUNER, Edward M.

1991: Transformation of Self in Tourism. In: Annals of Tourism Research 18, S. 238-250.

BRUSATTI, Alois

1984: Hundert Jahre österreichischer Fremdenverkehr. Historische Entwicklung 1884-1984. Wien.

CANTAUW-GROSCHEK, Christiane

1993: „Liebe Kolleginnen und Kollegen ...“ Urlaubspostkarten an die Arbeitsstelle. In: Dieter KRAMER und Ronald LUTZ (Hg.): Tourismus-Kultur. Kultur-Tourismus. (= Kulturwissenschaftliche Horizonte 1) Hamburg S. 143-169.

EDER, Walter

1991: Zuhause in der Fremde? Der Verlust der Raumerfahrung als Verlust des Erfahrungsraumes beim Reisen. In: Ortfried SCHÄFFTER (Hg.), Das Fremde. Erfahrungsmöglichkeiten zwischen Faszination und Bedrohung. Opladen S. 158-172.

ENZENSBERGER, Hans Magnus

1962: Eine Theorie des Tourismus. In: ders.: Einzelheiten I. Bewußtseins-Industrie. Frankfurt/M. S. $147-168$.

FENDL, Elisabeth und LÖFFLER, Klara

1995: Die Reise im Zeitalter ihrer technischen Reproduzierbarkeit: zum Beispiel Diaabend. In: Christiane CANTAUw (Hg.), Arbeit, Freizeit, Reisen. Die feinen Unterschiede im Alltag. (3. Arbeitstagung der DGV-Kommission Tourismusforschung von 23. bis zum 25. März. Münster/New York S. 55-68.

FIELHAUER, Helmut Paul

1982: Festland Österreich? Kritische Anmerkungen zur Kultur des Tourismus. In: Olaf BOCKHORN u.a. (Hg), Kulturjahrbuch 1. Wiener Beiträge zu Kulturwissenschaft und Kulturpolitik. Wien. S. 316-321.

Fremdenverkehr, Der in Österreich im Jahre 1991

1992: (= Beiträge zur österreichischen Statistik H. 1053) Wien.

GERAMB, Viktor

1946: Um Österreichs Volkskultur. Salzburg.

GÖTTSCH, Silke

1994: Frühe Tourismuskritik in der Heimatschutzbewegung. In: Burkhard PÖTTLER und Ulrike KAMMERHOFER-AGGERMANN, Tourismus und Regionalkultur. Referate der Österreichischen Volkskundetagung 1992 in Salzburg. (= Buchreihe der Österreichischen Zeitschrift für Volkskunde Bd 12) Wien, S. 25-40.

GREVERUS, Ina Maria

1978: Tourismus und interkulturelle Kommunikation. In: Zeitschrift für Kulturaustausch. Jg. 28 (,Tourismus und Kulturwandel“), S. 96-107.

Greverus, Ina Maria, Köstlin, Konrad und SCHILling, Heinz

1988: Kulturkontakt-Kulturkonflikt. Zur Erfahrung des Fremden (Notizen Bd 28). Frankfurt/M.

GYR, Ueli

1988: Touristenkultur und Reisealltag. Volkskundlicher Nachholbedarf in der Tourismusforschung. In: Zeitschrift für Volkskunde 84. S. 224-239.

1992: Kultur für Touristen und Touristenkultur. Plädoyer für qualitative Analysen in der Reiseforschung. In: KRAMER/LUTZ S. 19-38.

1994: Touristenverhalten und Symbolstrukturen. Zur Typik des organisierten Erlebniskonsums. In: Burkhard PÖTTLER und Ulrike KAMMERHOFER-AGGERMANN, Tourismus und Regionalkultur. Referate der Osterreichischen Volkskundetagung 1992 in Salzburg. (= Buchreihe der Osterreichischen Zeitschrift für Volkskunde Bd 12) Wien, S. 41-56.

HAHN, Heinz und KAGELMANN H. Jürgen (Hg.)

1993: Tourismuspsychologie und Tourismussoziologie. Ein Handbuch zur Tourismuswissenschaft. München. 
HAID, Hans

1988: Tourismus als Stress und Störfaktor. Soziokulturelle Folgen für die Bevölkerung in den ZielRegionen und die Nicht-Diskussion entsprechender Fragen in tourismusabhängigen Regionen. In: GREVERUS u.a., 1988, S. 357-363.

HOCHSCHILD, Arlie Russell

1990: Das gekaufte Herz. Zur Kommerzialisierung der Gefühle. (= Theorie und Gesellschaft Bd 13) Frankfurt, New York.

HofMANN, Gabriele (Hg.)

1994: Über den Zaun geguckt. Freizeit auf dem Dauercampingplatz und in der Kleingartenanlage. (= Kulturanthropologie-Notizen 45) Frankfurt.

HÖRANDNER, Edith und LUNZER, Hans (Hg.)

1982: Folklorismus. Vorträge der 1. internationalen Arbeitstagung des Vereins „Volkskultur um den Neusiedlersee" in Neusiedl/See 1978. (= Neusiedler Konfrontationen 1) Neusiedl/See.

HUNZIKER, Walter und KRAPF, Kurt

1942: Grundriß der Allgemeinen Fremdenverkehrslehre. Zürich.

JEDAMSKY, Doris, JEHLE, Hiltgung und SiEBERT, Ulla (Hg.)

1994: „... und tät das Reisen wählen. Beiträge zur Frauenreiseforschung. Bern.

JEGGLE, Utz und KORFF, Gottfried

1974: Zur Entwicklung des Zillertaler Regionalcharakters. Ein Beitrag zur Kulturökonomie. In: Zeitschrift für Volkskunde. Jg. 70, S. 39-57.

JOHLER, Reinhard

1994: Resistance through Rituals: Eine Lokalstudie zum Tourismus im Kleinen Walsertal/Vorarlberg. In: Burkhard PÖTTLER und Ulrike KAMMERHOFER-AGgERMANN, Tourismus und Regionalkultur. Referate der Österreichischen Volkskundetagung 1992 in Salzburg. (= Buchreihe der Österreichischen Zeitschrift für Volkskunde Bd 12) Wien, S. 239-258.

KAGELMANN, H. Jürgen (Hg.)

1993: Tourismuswissenschaft. Soziologische, sozialpsychologische und sozialanthropologische Untersuchungen. München.

KAMMERHOFER-AGGERMANN, Ulrike und SCOPE, Alma

1994: Kleidungsverhalten von Künstlern und Gästen der Salzburger Festspiele und der einheimischen Bevölkerung zwischen 1920 und 1938 - eine Wechselwirkung? In: Burkhard PöTTLER und Ulrike KAMMERHOFER-AGGERMANN, Tourismus und Regionalkultur. Referate der Österreichischen Volkskundetagung 1992 in Salzburg. (= Buchreihe der Osterreichischen Zeitschrift für Volkskunde Bd 12) Wien, S. 149-161.

KAPELLER, Kriemhild

1991: Tourismus und Volkskultur. Folklorismus - zur Warenästhetik der Volkskultur. Ein Beitrag zur alpenländischen Folklorismusforschung am Beispiel des Vorarlberger Fremdenverkehrs mit besonderer Berücksichtigung der Regionen Montafon und Bregenzerwald. (= Dissertationen der Karl-Franzens-Universität Graz, Bd 81) Graz.

KERSCHBAUMER, Gert

1994: Tourismus im politischen Wandel der 30er und 40er Jahre. In: Hanns HAAS, Robert HofFMANN und Kurt LUGER (Hg.), Weltbühne und Naturkulisse. Zwei Jahrhunderte SalzburgTourismus. Salzburg, S. 120-128.

KNEBEL, Hans-Joachim

1960: Soziologische Strukturwandlungen im modernen Tourismus. (= Soziologische Gegenwartsfragen N. F. 8) Stuttgart.

KöSTLIN, Konrad

1991: Souvenir. Das kleine Geschenk als Gedächtnisstütze. In: Übriges. Kopflose Beiträge zu einer volkskundlichen Anatomie. Utz Jeggle zum 22. Juni 1991. Tübingen, S. 131-141.

1994: Reisen, regionale Kultur und die Moderne. Wie die Menschen modern wurden, das Reisen lernten und dabei die Region entdeckten. In: Burkhard PÖTTLER und Ulrike KAMMERHOFERAGGERMAnN, Tourismus und Regionalkultur. Referate der Österreichischen Volkskundetagung 1992 in Salzburg. (= Buchreihe der Österreichischen Zeitschrift für Volkskunde Bd 12) Wien, S. 11-24.

1995: Wir sind alle Touristen - Gegenwelten als Alltag. In: Christiane CANTAUW (Hg.), Arbeit, Freizeit, Reisen. Die feinen Unterschiede im Alltag. (3. Arbeitstagung der DGV-Kommission Tourismusforschung von 23. bis zum 25. März. Münster/New York, S. 1-12. 
KRAJICEK, Helmut

1979: Die Ausseer Tracht. In: ÖZV 82, S. 42-60.

KRAMER, Dieter

1983: Der sanfte Tourismus. Umwelt- und sozialverträglicher Tourismus in den Alpen. Wien.

1990: Tourismus-Politik. Aufsätze aus 12 Jahren Tourismus-Diskussion. Münster.

1992: Kulturwissenschaftliche Tourismus-Forschung. In: Dieter KRAMER und Ronald LUTZ (Hg.):

Reisen und Alltag. Beiträge zur kulturwissenschaftlichen Tourismusforschung. (Notizen Bd 39) Frankfurt/M., S. 11-17.

KRIPPENDORF, Jost, ZIMMER, Peter und GLAUBER, Hans

1988: Für einen anderen Tourismus. Probleme-Perspektiven-Ratschläge. Fankfurt/M.

KULINAT, Klaus und STEINECKE, Albrecht

1984: Geographie des Freizeit- und Fremdenverkehrs. (= Erträge der Forschung Bd 212) Darmstadt.

LAUTERBACH, Burkhart

1989: Baedeker und andere Reiseführer. Eine Problemskizze. In: Zeitschrift für Volkskunde 85, S. 206-234.

1993: Thesen zur kulturwissenschaftlichen Reiseführer-Forschung. In: Dieter KRAMER und Ronald LUTZ (Hg.), Reisen und Alltag. Beiträge zur kulturwissenschaftlichen Tourismusforschung. (= Notizen Bd 39) Frankfurt/M. S. 55-69.

LIPP, Franz

1972: „Selbstdarstellung“ in der Volkskunst. Ein Beitrag zum Folklorismusproblem. In: Klaus BEITL (Hg.), Volkskunde. Fakten und Analysen. Festgabe für Leopold Schmidt zum 60. Geburtstag. Wien, S. 426-432.

LÖFGREN, Orvar

1994: Learning to Be a Tourist. In: Ethnologia Scandinavica 24, S. 102-125.

1986: Wish You Were Here! Holiday Images and Picture Postcards. In: Nils-Arvid BRINGÉUS (Hg.): Man and picture. Papers from the 1. International Symposium für Ethnographic Picture Research in Lund. Stockholm, S. 90-107.

MACCANNELL, Dean

1989: The Tourist. A New Theory of the Leisure Class. New York (2.Aufl.)

MEIXNER, Wolfgang

1994: „...dass es etwas gar zu viel Cultur in die Berge bringt.“ - Aspekte der Bewertung des frühen Fremdenverkehrs in Tirol durch Gäste und Einheimische. In: Burkhard PöTTLER und Ulrike KAMMERHOFER-AGGERMANN. Tourismus und Regionalkultur. Referate der Österreichischen Volkskundetagung 1992 in Salzburg. (= Buchreihe der Österreichischen Zeitschrift für Volkskunde Bd 12) Wien, S. 129-147.

MENASSE, Robert

1993: Das Land ohne Eigenschaften. Essay zur österreichischen Identität. Wien.

MOSER, Hans

1962: Vom Folklorismus in unserer Zeit. In: Zeitschrift für Volkskunde 58. S. 177-209.

1964: Der Folklorismus als Forschungsproblem der Volkskunde. In: Hessische Blätter für Volkskunde 55, S. 9-57.

MÜLLER, Robert

1984: Fremdenverkehrswerbung in Österreich. Historische Beispiele seit 1884. Wien.

NUSSBAUMER, Josef

1988: Die „Tausend-Mark-Sperre“ vom Mai 1933 und der Tiroler Fremdenverkehr. In: Thomas ALBRICH, Klaus EISTERER und Rolf STEININGER (Hg.), Tirol und der Anschluß. Voraussetzungen, Entwicklungen, Rahmenbedingungen 1918-1938. Innsbruck, S. 307-330.

OBERHAMMER, Monika

1983: Sommervillen im Salzkammergut. Die spezifische Sommerfrischenarchitektur des Salzkammergut in der Zeit von 1830 bis 1918. Salzburg.

OPASCHOWSKI, Horst W.

1989: Tourismusforschung. (= Freizeit- und Tourismusstudien 3) Opladen.

PRAHL, Hans-Werner und STEINECKE, Albrecht

1989: Der Millionen-Urlaub. Von der Bildungsreise zur totalen Freizeit. Darmstadt und Neuwied.

Preglau, Max, Meleghy, Tamas, Frantz, Klaus und TAFERTSHOFER, Alois

1985: Fremdenverquer. Kosten und Nutzen des Tourismus am Beispiel Obergurgl (= Schriftenreihe der Michael-Gaismair-Gesellschaft Bd 4), Innsbruck. 
Reisegewohnheiten der Österreicher im Jahre 1990.

1992: Ergebnisse des Mikrozensus Dezember 1990. (= Beiträge zur österreichischen Statistik H. 1065) Wien.

ROMEIS-STRACKE, Felizitas

1995: Service-Qualität im Tourismus. Grundsätze und Gebrauchsanweisungen für die touristische Praxis. (Hg. Allgemeiner Deutsche Automobil-Club e.V.) München.

SCHARFE, Martin

1991: Die alte Straße. Fragmente. In: BAUSINGER/BEYRER/KorFF, S. 11-22

SCHIVELBUSCH, Wolfgang

1989: Geschichte der Eisenbahnreise. Zur Industrialisierung vom Raum und Zeit im 19. Jahrhundert. Frankfurt/M.

SCHÖNBERGER, Alwin

1994: Alm-Rausch. Die Tragödie hinter der Freizeitmaschinerie. Wien.

SCHRUTKA-RECHTENSTAMM, Adelheid

1992: Beobachtungen und Überlegungen zu neuen Tendenzen des „Urlaubs am Bauernhof“. In: Dieter KRAMER und Ronald LUTZ (Hg.): Reisen und Alltag. Beiträge zur kulturwissenschaftlichen Tourismusforschung. (Notizen Bd 39) Frankfurt/M. S. 131-145.

1994: „Die Gäste fühlen sich wohl bei uns“ - Begegnungen durch Tourismus. In: Burkhard PÖTTLER und Ulrike KAMMERHOFER-AGgERMANN, Tourismus und Regionalkultur. Referate der Österreichischen Volkskundetagung 1992 in Salzburg. (= Buchreihe der Österreichischen Zeitschrift für Volkskunde Bd 12) Wien, S. 85-94.

1995: Einflüsse des Fremdenverkehrs auf die Alltagskultur im Alpenraum. In: Christiane CANTAUW (Hg.), Arbeit, Freizeit, Reisen. Die feinen Unterschiede im Alltag. (3. Arbeitstagung der DGVKommission Tourismusforschung von 23. bis zum 25. März. Münster/New York, S. 151-160.

1996: Die Symbolik der Gastfreundschaft im Tourismus. In: Klaus WEIERMAIR (Hg.), Alpine Tourism. Sustainability: Reconsidered and Redisigned. Proceedings of the International Conference at the University if Innsbruck May 1996. Innsbruck. S. 354-363

1997a: „Wo Alltag zum Erlebnis wird“. Konzepte des Bauernhofurlaubs im europäischen Vergleich. In: Josef VAR̆EKA, Markéta HolubOVA, Lydia PETRÁNOVÁ (Hg.), Evropský kulturní prostor - jednota v rozmanitosti. Europäischer Kulturraum - Einheit in der Vielfalt. European Cultural Area - Unity in Diversity. Sborník z 10. etnokartografického sympozia Trešt. Referate der 10. Internationalen Tagung der europäischen ethnokartographischen Arbeitsgruppe. Praha. S. 222-229.

1997b: Zur Entstehung und Bedeutung von Symbolen im Tourismus. In: Rolf Wilhelm BREDNICH und Heinz SchMiTT (Hg.), Symbole. Zur Bedeutung der Zeichen in der Kultur. 30. Deutscher Volksundekongreß in Karlsruhe. Münster u. a. S. 123-134.

1997c: Gäste und Gastgeber: touristische Ritualisierungen diesseits und jenseits der Bezahlung. In: Tourismus Journal. Jg. 1, Heft 3/4, S. 467-481.

1998: Die Rolle der Gastgeber: Touristische Gastfreundschaft als kulturelles und wirtschaftliches Prinzip. In: Salzburger Volkskultur. Jg. 22, S. 39-48.

SCHWEIGER, Günte

1992: Österreichs Image in der Welt. Ein weltweiter Vergleich mit Deutschland und der Schweiz. Wien.

SEITZ, Gabriele

1987: Wo Europa den Himmel berührt. Die Entdeckung der Alpen. München.

SHAMIR, Boas

1993: Zwischen Dankbarkeit und Verpflichtung. Eine Analyse des Trinkgeldgebens. In: KAGELMANN, H. Jürgen (Hg.): Tourismuswissenschaft. Soziologische, sozialpsychologische und sozialanthropologische Untersuchungen. München, S. 97-114.

SOEFFNER, Hans-Georg

1991: Zur Soziologie des Symbols und des Rituals. In: Jürgen OelKers, Klaus WegEnAST (Hg.), Das Symbol - Brücke des Verstehens. Stuttgart, Berlin, Köln, S. 63-81.

STAGL, Justin

1983: Apodemiken. Eine räsonierte Bibliographie der reisetheoretischen Literatur des 16., 17. und 18. Jahrhunderts (= Quellen und Abhandlungen zur Geschichte der Staatsbeschreibung und Statistik Bd 2). Paderborn-München-Wien-Zürich.

STEINER, Gertraud

1987: Die Heimatmacher. Kino in Österreich 1946-1966. Wien. 


\section{THIEM, Marion}

1994: Tourismus und kulturelle Identität. Die Bedeutung des Tourismus für die Kultur touristischer Ziel- und Quellgebiete. Bern und Hamburg.

TSCHOFEN, Bernhard

1994: Die Seilbahnfahrt. Gebirgswahrnehmung zwischen klassischer Alpenbegeisterung und moderner Ästhetik. In: Burkhard PÖTTLER und Ulrike KAMMERHOFER-AGGERMANN, Tourismus und Regionalkultur. Referate der Österreichischen Volkskundetagung 1992 in Salzburg. (= Buchreihe der Österreichischen Zeitschrift für Volkskunde Bd 12) Wien, S. 107-127.

URRY, John

1990: The Tourist Gaze, Leisure and Travel in Contemporary Societies. London.

VESTER, Heinz-Günter

1993: Tourismussoziologie. In: HAHN/KAGELMANN, S. 36-43.

WAHRLICH, Heide

1984: Tourismus - eine Herausforderung für Ethnologen. Problemdimensionen und Handlungsaspekte im touristischen Bezugsfeld. Berlin.

WEISS, Richard

1946: Volkskunde der Schweiz. Erlenbach/Zürich.

1962: Alpiner Mensch und alpines Leben in der Krise der Gegenwart. In: Schweizer. Archiv f. Vk., Jg. 58, S. 232-254.

WEYERS, Dorle und KÖCK, Christoph

1995: Mit Abdulla durch die Welt und mit Birkel zum Mond. Zum kulturellen Sinn des Werbemediums Sammelbild. In: Christiane CANTAUw (Hg.), Arbeit, Freizeit, Reisen. Die feinen Unterschiede im Alltag. (3. Arbeitstagung der DGV-Kommission Tourismusforschung von 23.

WoOD, Roy C bis zum 25. März. Münster/New York, S. 21-40.

1994: Hotel Culture and Social Control. In: Annals of Tourism Research 21. S. 65-80

Zeitschrift für österreichische Volkskunde

1895: Jg. 1, S. 121f.

ZIMMERMANN, Friedrich

1987: Aktuelle Tendenzen des Tourismus in den österreichischen Alpen. In: Z. f. Wirtschaftsgeographie 31 , S. 106-117. 